\title{
THE EFFECT OF SALT DEPRIVATION ON THE URINARY CONCENTRATING MECHANISM IN THE DOG*
}

\author{
By CARL GOLDSMITH, $\dagger$ H. KENT BEASLEY, PEGGY J. WHALLEY, $\dagger$ \\ FLOYD C. RECTOR, JR. AND DONALD W. SELDIN
}

\author{
(From The Department of Internal Medicine, The University of Texas Southwestern Medical \\ School, Dallas, Texas)
}

(Submitted for publication June 8, 1961 ; accepted August 4, 1961)

According to the countercurrent theory (1-3), concentration of the urine results from the movement of water from the collecting tubule into the hypertonic medullary interstitium. Medullary hypertonicity is generated and maintained by the sodium transported out of the ascending limb of the loop of Henle and trapped in the medulla by the vasa recta which form a countercurrent exchanger. Consequently, the availability of sodium for reabsorption in the loop of Henle may assume a critical role in the regulation of urinary concentration.

Various derangements in water excretion have been detected when the intake or renal excretion of sodium is altered. Patients with congestive heart failure, cirrhosis of the liver, and other conditions characterized by diminished sodium excretion frequently have been reported to have impaired urinary concentration in the absence of intrinsic renal disease. Levinsky, Davidson and Berliner (4) noted that dogs maintained on a sodium-free diet showed decreased maximal urinary concentration. Levitt, Levy and Polimeros (5), however, found that salt restriction in normal human subjects for 5 days had no effect on maximal urinary concentration.

The present studies were undertaken to examine the relationship of sodium metabolism to urinary concentrating ability by evaluating the effects of varying sodium intake, glomerular filtration rate (GFR), aldosterone activity, and serum

\footnotetext{
* Supported in part by a grant from The National Institutes of Health, and in part by a grant from the Dallas Heart Association. This work has been presented in part before the meeting of the Southern Section, American Federation for Clinical Research, New Orleans, January 19, 1961, and in part before the meeting of the American Federation for Clinical Research, Atlantic City, April 30, 1961.

† Work done as a Public Health Service trainee of The National Institutes of Health.
}

sodium concentration on renal water conservation in dogs, as reflected by their capacity to elaborate a maximally concentrated urine and to form $\mathrm{T}^{\mathrm{c}} \mathrm{H}_{2} \mathrm{O}$.

\section{METHODS}

Female mongrel dogs weighing 8 to $18 \mathrm{~kg}$ were used. Each dog was tube-fed once daily an artificial diet that provided $4.3 \mathrm{mEq}$ potassium, $1.1 \mathrm{~g}$ protein, 27 calories, and $30 \mathrm{ml}$ water per $\mathrm{kg}$ of body weight per day. When access to sodium was permitted, $1.5 \mathrm{mEq}$ sodium per $\mathrm{kg}$ body weight per day was added to the diet, whereas the sodium-free diet provided less than $0.02 \mathrm{mEq}$ sodium per kg body weight per day. The amount of diet fed any given dog was kept constant.

Acute experiments were performed to evaluate maximum urinary concentrating ability and the maximum rate of $\mathrm{T}^{\mathrm{c}} \mathrm{H}_{2} \mathrm{O}$ formation. Food and water were withheld for the 24 hours preceding the experiment. Two hours prior to study, $5 \mathrm{U}$ of vasopressin (Pitressin tannate in oil) was administered i.m. At the time of the study, dogs were lightly anesthetized with sodium pentobarbital, and bladder urine was obtained at once for the determination of maximum urinary concentration. To measure $\mathrm{T}^{\mathrm{c}} \mathrm{H}_{2} \mathrm{O}$ formation, 10 per cent mannitol in 5 per cent dextrose in water was infused intravenously at a rate of approximately $1 \mathrm{ml}$ per minute during the 20 -minute equilibration period; subsequently the rate was increased in stepwise fashion to induce graded solute diuresis. In accordance with the suggestion of Giebisch and Lozano (6), 75 mmoles of sodium chloride was added to each $\mathrm{L}$ of mannitol infusion to minimize undue washout of papillary sodium by osmotic diuresis alone (7). To assure sustained maximal levels of antidiuretic hormone (ADH), $33 \mathrm{mU}$ per $\mathrm{kg}$ body weight vasopressin was given with the inulin prime at the start of the equilibration period and thereafter constantly infused at a rate of $50 \mathrm{mU}$ per $\mathrm{kg}$ per hour in 5 per cent dextrose in water, adjusted to $\mathrm{pH} 5.5$ with acetic acid. Inulin was infused at a constant rate of $10 \mathrm{mg}$ per minute. Urine was allowed to drain freely from an indwelling bladder catheter. Collection periods were terminated by air washout and manual compression of the bladder. Arterial blood samples were drawn at midpoints of the urine collection periods into heparinized syringes. Osmolalities of serum and urine were determined on the Fiske osmometer. Inulin was measured by the resorcinol method of Schreiner 
(8). Sodium and potassium were determined with an internal standard flame photometer.

The effects of variations in salt intake, serum sodium concentration, and aldosterone on maximum urinary concentration and maximum $\mathrm{T}^{\mathrm{c}} \mathrm{H}_{2} \mathrm{O}$ formation were investigated in dogs treated as follows.

A) Sodium-fed control. Dogs received sodium-containing diet for 2 to 5 days.

B) Sodium-restricted. Dogs received sodium-free diet for 3 to 9 days.

C) Aldosterone and aldosterone antagonists. Dogs receiving sodium-containing diet were given DL-aldosterone ${ }^{1}$ in a dose of 30 to $500 \mu \mathrm{g}$ per $\mathrm{kg}$ body weight i.v. 2 hours prior to study. Dogs receiving sodium-free diet for 1 week were then maintained on this diet and a spirolactone was given for an additional week. One dog was given SC-9420, ${ }^{2} 100 \mathrm{mg}$ orally once daily; the other five were given SC-11929,3 10, 20, or $100 \mathrm{mg}$ i.m. once daily.

D) Raising GFR during sodium restriction. GFR was raised in sodium-restricted dogs by the administration of slowly released methylprednisolone (Depo-Medrol), $50 \mathrm{mg}$ i.m., every other day for 4 to 6 days, and regular methylprednisolone (Solu-Medrol), $40 \mathrm{mg}$ i.v., $20 \mathrm{~min}$ utes prior to onset of the study. One dog was given only the acute dose.

E) Hyponatremia. Hyponatremia was induced by the administration of $5 \mathrm{U}$ of vasopressin i.m. at $7 \mathrm{a.m}$. and 4 p.m. daily. These dogs received either the sodiumfree or sodium-containing diet as indicated and were tube-fed additional water to make a total of 4 to 6 per cent of body weight per day. Studies were done in 3 to 4 days depending on the degree of hyponatremia present.

At least 3 days elapsed between consecutive studies in any given dog. Since obligatory sodium loss during diuresis in sodium-deprived dogs averaged $20 \mathrm{mEq}$, this amount of sodium was given to all dogs with the next feeding after study. Free access to water was allowed until 48 hours prior to study to prevent dehydration, and i.m. iron was given to prevent iron deficiency.

\section{CALCULATIONS}

For purposes of comparison, results are discussed in terms of the regression of osmolar clearance ( $\mathrm{C}_{0} \Perp \mathrm{m}$ ) on flow in milliliters per minute (V). Cosm is defined as $\mathrm{U}_{\mathrm{Osm}} / \mathrm{P}_{\mathrm{Osm}} \times \mathrm{V}$. $\mathrm{T}^{\mathrm{c}} \mathrm{H}_{2} \mathrm{O}$ refers to water reabsorbed in excess of solute as measured from isosmotic urine, and is derived by the equation $\mathrm{T}^{\mathrm{c}} \mathrm{H}_{2} \mathrm{O}=\mathrm{C}_{\mathrm{osm}}-$ $\mathrm{V}$, after Zak, Brun and Smith (9). Slopes were obtained by the method of least squares from observations made at flows between 3 and $13 \mathrm{ml}$ per minute in which

1 Kindly supplied by Dr. Robert Gaunt, Ciba Inc., Summit, N. J.

2 3-(3-Oxo-7 $\alpha$-acetylthio-17 $\beta$-hydroxy-4-androskn-17 $\alpha$ yl) propionic acid-y-lactone-(Aldactone).

3 3-Oxo-9 $\alpha$-fluoro-11 $\beta$-dihydroxy-17 $\alpha$-pregn-4-ene-21carboxylic acid (K salt). Both SC-9420 and SC-11929 were kindly supplied by Dr. Clarence L. Gantt, G. D. Searle \& Co., Chicago, Ill. range the regression is linear. Since the slope of regression may vary widely from unity, maximum $\mathrm{T}^{\mathrm{c}} \mathrm{H}_{2} \mathrm{O}$ $\left(\mathrm{T}^{\mathrm{c}} \mathrm{H}_{2} \mathrm{O}\right)$ in this report was estimated from the single greatest observed $\mathrm{T}^{\mathrm{c}} \mathrm{H}_{2} \mathrm{O}$ corrected to $100 \mathrm{ml}$ GFR $\left[\mathrm{T}^{\mathrm{c}}{ }_{\mathrm{M}} \mathrm{H}_{2} \mathrm{O}=\left(\max \mathrm{T}^{\mathrm{c}} \mathrm{H}_{2} \mathrm{O} / \mathrm{GFR}\right) \times 100\right]$ rather than the usual value that represents the average $\mathrm{T}^{\mathrm{c}} \mathrm{H}_{2} \mathrm{O}$ over the linear range of the regression. Since the $\mathrm{T}^{\mathrm{c}} \mathrm{H}_{2} \mathrm{O}$ formation was not constant under certain experimental conditions, an expression was devised to compare $\mathrm{T}^{\mathrm{c}} \mathrm{H}_{2} \mathrm{O}$ at comparable urine flows corrected for variations in body size. This expression, referred to as $\mathrm{T}^{\mathrm{c}}{ }_{\mathrm{DH}} \mathrm{H}_{2} \mathrm{O}$, was calculated as that value of $\mathrm{T}^{\mathrm{c}} \mathrm{H}_{2} \mathrm{O}$ formed at a urine flow equal to one-half of the initial body weight in kilograms of a given dog and corrected to $100 \mathrm{ml} \mathrm{GFR}$. The point at which the regression line of $\mathrm{T}^{\mathrm{c}} \mathrm{H}_{2} \mathrm{O}$ intersected the isosmotic line was designated the crossover point, and represents the beginning of the formation of hypotonic urine. All statistical analyses were done by standard methods (10). The number of periods in each experiment varied from 4 to 15 , the usual being 6 or 7 . Collection periods usually lasted 10 minutes. Only studies in which GFR was relatively stable throughout are reported.

\section{RESULTS}

At the onset of this study, two sodium-fed trained dogs were studied supine and unanesthetized. Several days later they were restudied after being anesthetized in the manner described for all subsequent studies. In neither dog did anes-

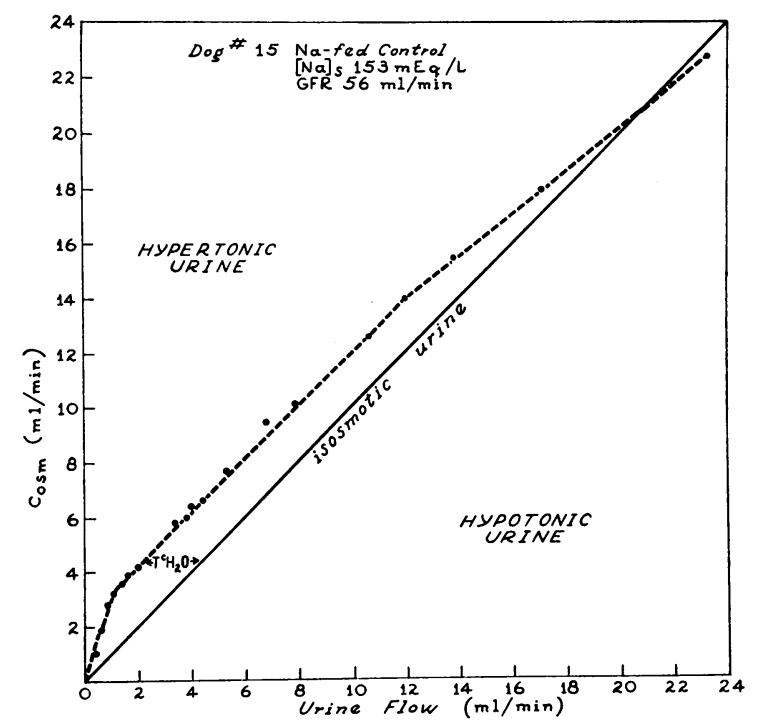

Fig. 1. The Formation of $\mathrm{T}^{\mathrm{e}} \mathrm{H}_{2} \mathrm{O}$ DURING ManNitol DIURESIS IN A SODIUM-FED CONTROL DOG. In this and subsequent figures, $\mathrm{C}_{\mathrm{osm}}$ and urine flow were not corrected to a standard GFR. Therefore, the values for $\mathrm{T}^{\circ} \mathrm{H}_{2} \mathrm{O}$ that can be derived from these figures are different from those listed in the tables, where a correction for GFR was made. 
TABLE I

The effects of varying salt intake on maximum urinary concentrating ability and $T^{\circ} \mathrm{H}_{2} \mathrm{O}$ formation during mannitol diuresis

\begin{tabular}{|c|c|c|c|c|c|c|c|c|c|c|}
\hline Dog & Preparation & Day & $\underset{\mathrm{Na}}{\text { Serum }}$ & $\underset{\mathbf{K}}{\text { Serum }}$ & GFR* & $\begin{array}{l}\text { Maximum } \\
\text { osmotic } \\
\text { U/P ratio }\end{array}$ & $\begin{array}{l}\begin{array}{l}\text { Maximum } \\
\text { observed } \\
\frac{\mathrm{T} \cdot \mathrm{H}_{2} \mathrm{O}}{\mathrm{GFR}} \times 100\end{array} \\
\end{array}$ & $\begin{array}{c}\text { Derived } \\
\frac{T^{\circ} \mathrm{H}_{2} \mathrm{O}}{\mathrm{GFR}} \times 100\end{array}$ & Slope & $\begin{array}{c}\text { Calculated } \\
\text { point of } \\
\text { crossovert }\end{array}$ \\
\hline & & & $m E q / L$ & $m E q / L$ & $\mathrm{ml} / \mathrm{min}$ & & $\begin{array}{l}m l / 100 \\
m l \text { GFR }\end{array}$ & $\begin{array}{l}m l / 100 \\
m l ~ G F R\end{array}$ & & $m l / m i n$ \\
\hline 14 & $\begin{array}{l}\text { Na-fed } \\
\text { Na-free }\end{array}$ & 7 & $\begin{array}{l}150 \\
156\end{array}$ & $\begin{array}{l}3.8 \\
5.2\end{array}$ & $\begin{array}{l}52 \\
26\end{array}$ & $\begin{array}{l}2.9 \\
2.8\end{array}$ & $\begin{array}{l}5.9 \\
3.5\end{array}$ & $\begin{array}{r}5.2 \\
-0.8\end{array}$ & $\begin{array}{l}1.04 \\
0.77\end{array}$ & $\begin{array}{r}>20 \\
4.3\end{array}$ \\
\hline 29 & $\begin{array}{l}\text { Na-fed } \\
\text { Na-free }\end{array}$ & 7 & $\begin{array}{l}157 \\
152\end{array}$ & $\begin{array}{l}3.4 \\
4.1\end{array}$ & $\begin{array}{l}70 \\
54\end{array}$ & $\begin{array}{l}3.3 \\
2.5\end{array}$ & $\begin{array}{l}5.0 \\
1.7\end{array}$ & $\begin{array}{r}4.2 \\
-1.3\end{array}$ & $\begin{array}{l}0.91 \\
0.72\end{array}$ & $>\underset{3.6}{20}$ \\
\hline 42 & $\begin{array}{l}\text { Na-fed } \\
\text { Na-free }\end{array}$ & 3 & $\begin{array}{l}152 \\
156\end{array}$ & $\begin{array}{l}2.9 \\
3.6\end{array}$ & $\begin{array}{l}42 \\
39\end{array}$ & $\begin{array}{l}4.3 \\
3.9\end{array}$ & $\begin{array}{l}5.8 \\
2.5\end{array}$ & $\begin{array}{l}4.4 \\
0.9\end{array}$ & $\begin{array}{l}0.98 \\
0.86\end{array}$ & $>20$ \\
\hline 43 & $\begin{array}{l}\text { Na-fed } \\
\text { Na-free }\end{array}$ & 6 & $\begin{array}{l}149 \\
144\end{array}$ & $\begin{array}{l}4.3 \\
4.6\end{array}$ & $\begin{array}{l}56 \\
37\end{array}$ & $\begin{array}{l}4.4 \\
3.9\end{array}$ & $\begin{array}{l}5.3 \\
1.3\end{array}$ & $\begin{array}{r}4.4 \\
-0.2\end{array}$ & $\begin{array}{l}0.95 \\
0.74\end{array}$ & $>\underset{5.4}{20}$ \\
\hline 47 & $\begin{array}{l}\text { Na-fed } \\
\text { Na-free }\end{array}$ & 7 & $\begin{array}{l}149 \\
152\end{array}$ & $\begin{array}{l}4.5 \\
5.3\end{array}$ & $\begin{array}{l}81 \\
66\end{array}$ & $\begin{array}{l}4.0 \\
2.8\end{array}$ & $\begin{array}{l}1.6 \\
0.8\end{array}$ & $\begin{array}{r}1.4 \\
-0.8\end{array}$ & $\begin{array}{l}1.02 \\
0.96\end{array}$ & $\gtrless 20$ \\
\hline 48 & $\begin{array}{l}\text { Na-fed } \\
\text { Na-free }\end{array}$ & 9 & $\begin{array}{l}148 \\
144\end{array}$ & $\begin{array}{l}4.5 \\
4.8\end{array}$ & $\begin{array}{l}50 \\
44\end{array}$ & $\begin{array}{l}4.0 \\
3.5\end{array}$ & $\begin{array}{l}6.5 \\
2.3\end{array}$ & $\begin{array}{l}4.8 \\
1.2\end{array}$ & $\begin{array}{l}1.06 \\
0.99\end{array}$ & $>{ }_{12.2}^{20}$ \\
\hline 52 & $\begin{array}{l}\text { Na-fed } \\
\text { Na-free }\end{array}$ & 6 & $\begin{array}{l}142 \\
137\end{array}$ & $\begin{array}{l}4.2 \\
4.4\end{array}$ & $\begin{array}{l}62 \\
55\end{array}$ & $\begin{array}{l}5.5 \\
4.3\end{array}$ & $\begin{array}{l}2.7 \\
2.8\end{array}$ & $\begin{array}{l}2.5 \\
0.9\end{array}$ & $\begin{array}{l}1.02 \\
0.91\end{array}$ & $\begin{array}{l}>20 \\
12.2\end{array}$ \\
\hline 39 & $\begin{array}{l}\text { Na-fed } \\
\text { Na-free }\end{array}$ & 7 & $\begin{array}{l}143 \\
142\end{array}$ & $\begin{array}{l}3.4 \\
3.7\end{array}$ & $\begin{array}{l}66 \\
55\end{array}$ & $\begin{array}{l}4.4 \\
4.0\end{array}$ & $\begin{array}{l}5.2 \\
4.0\end{array}$ & $\begin{array}{l}\mathbf{5 . 4} \\
\mathbf{3 . 4}\end{array}$ & $\begin{array}{l}1.20 \\
1.12\end{array}$ & $\begin{array}{l}>20 \\
>20\end{array}$ \\
\hline 58 & $\begin{array}{l}\text { Na-fed } \\
\text { Na-free }\end{array}$ & 7 & $\begin{array}{l}159 \\
163\end{array}$ & $\begin{array}{l}3.9 \\
4.1\end{array}$ & $\begin{array}{l}53 \\
29\end{array}$ & & $\begin{array}{l}4.8 \\
1.4\end{array}$ & $\begin{array}{r}3.8 \\
-0.1\end{array}$ & $\begin{array}{l}1.07 \\
0.94\end{array}$ & $>\underset{6.7}{20}$ \\
\hline 62 & $\begin{array}{l}\text { Na-fed } \\
\text { Na-free }\end{array}$ & 7 & $\begin{array}{l}149 \\
143\end{array}$ & $\begin{array}{l}3.8 \\
4.7\end{array}$ & $\begin{array}{l}64 \\
60\end{array}$ & $\begin{array}{l}4.1 \\
3.2\end{array}$ & $\begin{array}{l}1.7 \\
0.5\end{array}$ & $\begin{array}{l}1.5 \\
0.6\end{array}$ & $\begin{array}{l}0.94 \\
0.97\end{array}$ & $\begin{array}{l}>20 \\
16.7\end{array}$ \\
\hline 66 & $\begin{array}{l}\text { Na-fed } \\
\text { Na-free }\end{array}$ & 6 & $\begin{array}{l}142 \\
143\end{array}$ & $\begin{array}{l}4.5 \\
4.7\end{array}$ & $\begin{array}{l}73 \\
54\end{array}$ & $\begin{array}{l}4.0 \\
4.0\end{array}$ & $\begin{array}{l}3.8 \\
3.1\end{array}$ & $\begin{array}{l}3.7 \\
1.6\end{array}$ & $\begin{array}{l}0.99 \\
0.75\end{array}$ & $\begin{array}{l}>20 \\
\quad 11.6\end{array}$ \\
\hline 67 & $\begin{array}{l}\text { Na-fed } \\
\text { Na-free }\end{array}$ & 6 & $\begin{array}{l}143 \\
141\end{array}$ & $\begin{array}{l}4.5 \\
4.0\end{array}$ & $\begin{array}{l}45 \\
41\end{array}$ & $\begin{array}{l}4.5 \\
3.6\end{array}$ & $\begin{array}{l}4.3 \\
1.6\end{array}$ & $\begin{array}{l}4.1 \\
1.3\end{array}$ & $\begin{array}{l}0.95 \\
0.89\end{array}$ & $>20.1$ \\
\hline \multicolumn{2}{|c|}{$\begin{array}{l}\text { Na-fed control } \\
\text { Mean } \\
\text { SD }\end{array}$} & & $\begin{array}{l}149 \\
\pm 1.8\end{array}$ & $\begin{array}{r}4.0 \\
\pm 0.5\end{array}$ & $\begin{array}{c}60 \\
\pm 12\end{array}$ & $\begin{array}{r}4.1 \\
\pm 0.7\end{array}$ & $\begin{array}{r}4.4 \\
\pm 1.6\end{array}$ & $\begin{array}{r}4.1 \\
\pm 1.1\end{array}$ & $\begin{array}{r}1.01 \\
\pm 0.08\end{array}$ & $>20$ \\
\hline \multicolumn{3}{|c|}{$\begin{array}{l}\text { Na-free } \\
\text { Mean }\end{array}$} & $\begin{array}{l}148 \\
\pm 2.5\end{array}$ & $\begin{array}{r}4.4 \\
\pm 0.5\end{array}$ & $\begin{array}{l}46 \\
\pm 13\end{array}$ & $\begin{array}{r}3.5 \\
\pm 0.6\end{array}$ & $\begin{array}{r}2.1 \\
\pm 1.1\end{array}$ & $\begin{array}{r}0.6 \\
\pm 1.2\end{array}$ & $\begin{array}{r}0.88 \\
\pm 0.12\end{array}$ & $\begin{array}{r}9.0 \\
\pm 4.6\end{array}$ \\
\hline \multicolumn{2}{|c|}{$\begin{array}{l}\text { Difference } \\
\text { Mean } \\
\mathbf{p}\end{array}$} & & $\begin{array}{l}-0.8 \\
>0.500\end{array}$ & & $\begin{array}{l}-14 \\
<0.001\end{array}$ & $\begin{array}{l}-0.6 \\
<0.001\end{array}$ & $\begin{array}{l}-2.3 \\
<0.001\end{array}$ & $<0.001$ & $\begin{array}{l}-0.12 \\
<0.001\end{array}$ & \\
\hline
\end{tabular}

* The glomerular filtration rate (GFR) represents the mean value for all collection periods. GFR remained relatively constant throughout $\dagger$ This figure represents the intersection of the regression line with the isosmotic line. At this urine flow, the urine would become hypotonic.
all studies reporte.

thesia depress $\mathrm{T}^{\mathrm{c}} \mathrm{H}_{2} \mathrm{O}$ formation. , Nevertheless, to minimize possible effects of anesthesia on maximal urinary concentration, only bladder urine formed before the animals were anesthetized was used for this measurement.

A. Sodium-fed control. Sodium-fed controls under the conditions of water restriction and exogenous $A D H$ had maximum osmotic $U / P$ ratios of $4.4 \pm 1.1$. During mannitol diuresis $\mathrm{T}^{\mathrm{c}} \mathrm{H}_{2} \mathrm{O}$ formation progressively increased until at moderate urine flows maximum values were attained. $\mathrm{T}^{\mathrm{c}} \mathrm{H}_{2} \mathrm{O}$ then remained relatively constant as urine flow was further increased (11). At urine flows between 3 and $13 \mathrm{ml}$ per minute, the regression of $\mathrm{C}_{\mathrm{Osm}}$ on $\mathrm{V}$ was linear (Figure 1). In 30 control ex- periments the mean slope of the regression was $1.00 \pm 0.08$; the $\mathrm{T}^{\mathrm{c}} \mathrm{MH}_{2} \mathrm{O}$ was $4.6 \pm 1.5 \mathrm{ml}$ per minute ; and the $\mathrm{T}_{\mathrm{D}} \mathrm{DH}_{2} \mathrm{O}$ was $3.9 \pm 1.4 \mathrm{ml}$ per minute. These figures are in agreement with some published control values in the $\operatorname{dog}(6,12)$, but $\mathrm{T}_{\mathrm{M}} \mathrm{H}_{2} \mathrm{O}$ and maximum osmotic $\mathrm{U} / \mathrm{P}$ ratios are lower than some others $(13,14)$. This is probably the result of a lower protein intake in our animals $(15,16)$.

At urine flows greater than $15 \mathrm{ml}$ per minute, the slope decreased sharply. In no instance, however, did a sodium-fed dog elaborate hypotonic urine at a urine flow less than $20 \mathrm{ml}$ per minute. This phenomenon has been pointed out by Raisz, $\mathrm{Au}$ and Scheer (17). 
B. Sodium restricted. Maximum urinary concentration and $\mathrm{T}^{\mathrm{c}} \mathrm{H}_{2} \mathrm{O}$ formation were determined in 12 dogs during periods of sodium feeding and then after salt restriction (Table I). Thus, each dog served as its own control. Salt restriction resulted in a mean fall in GFR of 23 per cent $(p<0.001)$ from control values. Maximum osmotic $\mathrm{U} / \mathrm{P}$ ratios diminished slightly (from 4.1 to $3.5)$ but significantly $(\mathrm{p}<0.001) . \quad \mathrm{T}_{\mathrm{M}}^{\mathrm{c}} \mathrm{H}_{2} \mathrm{O}$, in contrast, fell dramatically from 4.4 to $2.1 \mathrm{ml}$ per minute $(\mathrm{p}<0.001)$, while $\mathrm{T}_{\mathrm{D}}^{\mathrm{c}} \mathrm{H}_{2} \mathrm{O}$ fell from a control value of 4.1 to $0.6 \mathrm{ml}$ per minute. The slope diminished from 1.0 to 0.88 ( $p<0.001)$, so that frequently at moderate urine flows a hypotonic urine resulted (Figure 2). There was no change in the serum concentration of either sodium or potassium.

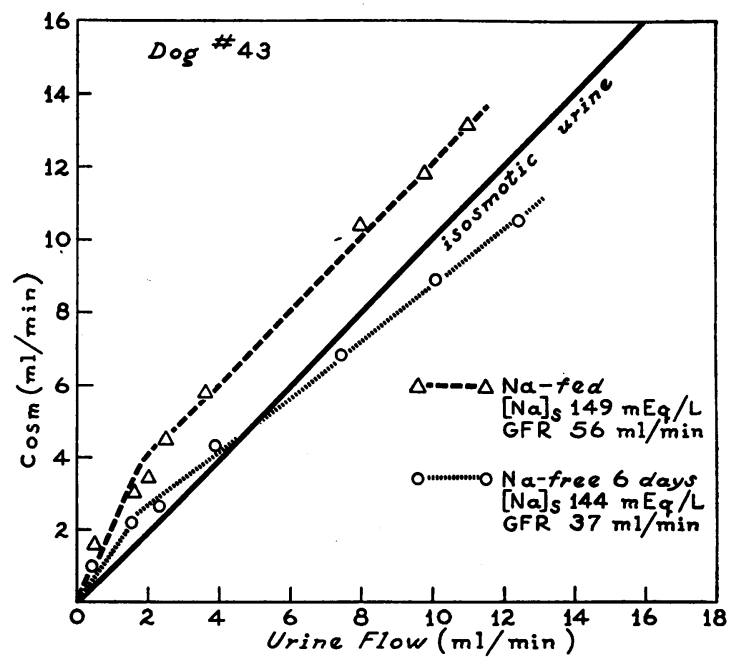

Fig. 2. The effect of SOdium depletion on $\mathrm{T}^{\mathrm{e}} \mathrm{H}_{2} \mathrm{O}$ FORMATION DURING MANNITOL DIURESIS.

TABLE II

The effects of varying doses of exogenous aldosterone on maximum urinary concentrating ability and $\mathrm{T}^{\mathrm{c}} \mathrm{H}_{2} \mathrm{O}$ formation during mannitol diuresis

\begin{tabular}{|c|c|c|c|c|c|c|c|c|c|c|}
\hline Dog & Preparation & Dose & $\underset{\mathbf{N a}}{\text { Serum }}$ & $\underset{\mathbf{K}}{\text { Serum }}$ & GFR & $\begin{array}{l}\text { Maximum } \\
\text { osmotic } \\
\text { U/P ratio }\end{array}$ & 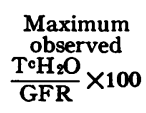 & $\begin{array}{c}\begin{array}{c}\text { Derived } \\
\text { To } \mathrm{H}_{2} \mathrm{O}\end{array} \times 100 \\
\text { GFR }\end{array}$ & Slope & $\begin{array}{l}\text { Calculated } \\
\text { point of } \\
\text { crossover }\end{array}$ \\
\hline & & $\begin{array}{c}\mu g / k g \\
b o d y \\
b t\end{array}$ & $m E q / L$ & $m E q / L$ & $m l / m i n$ & & $\begin{array}{l}m l / 100 \\
m l G F R\end{array}$ & $\begin{array}{l}m l / 100 \\
m l G F R\end{array}$ & & $m l / m i n$ \\
\hline 39 & $\begin{array}{l}\text { Control } \\
\text { Aldo. } \\
\text { Aldo. }\end{array}$ & $\begin{array}{l}30 \\
60\end{array}$ & $\begin{array}{l}145 \\
150 \\
140\end{array}$ & $\begin{array}{l}3.3 \\
3.9 \\
4.2\end{array}$ & $\begin{array}{l}67 \\
62 \\
72\end{array}$ & $\begin{array}{l}4.4 \\
3.5 \\
4.2\end{array}$ & $\begin{array}{l}3.2 \\
3.9 \\
2.6\end{array}$ & $\begin{array}{l}3.0 \\
3.2 \\
2.4\end{array}$ & $\begin{array}{l}1.03 \\
0.93 \\
0.93\end{array}$ & $\begin{array}{l}>20 \\
>20 \\
>20\end{array}$ \\
\hline 55 & $\begin{array}{l}\text { Control } \\
\text { Aldo. }\end{array}$ & 42 & $\begin{array}{l}146 \\
156\end{array}$ & $\begin{array}{l}4.2 \\
4.0\end{array}$ & $\begin{array}{l}40 \\
37\end{array}$ & $\begin{array}{l}5.3 \\
5.1\end{array}$ & $\begin{array}{l}4.0 \\
3.6\end{array}$ & $\begin{array}{l}2.2 \\
2.7\end{array}$ & $\begin{array}{l}1.00 \\
0.99\end{array}$ & $\begin{array}{l}>20 \\
>20\end{array}$ \\
\hline 56 & $\begin{array}{l}\text { Control } \\
\text { Aldo. }\end{array}$ & 36 & $\begin{array}{l}146 \\
155\end{array}$ & $\begin{array}{l}3.9 \\
4.2\end{array}$ & $\begin{array}{l}55 \\
54\end{array}$ & $\begin{array}{l}4.3 \\
6.3\end{array}$ & $\begin{array}{l}3.1 \\
7.4\end{array}$ & $\begin{array}{l}2.3 \\
6.1\end{array}$ & $\begin{array}{l}1.02 \\
1.12\end{array}$ & $\begin{array}{l}>20 \\
>20\end{array}$ \\
\hline 62 & $\begin{array}{l}\text { Control } \\
\text { Aldo. }\end{array}$ & 410 & $\begin{array}{l}149 \\
140\end{array}$ & $\begin{array}{l}3.8 \\
4.6\end{array}$ & $\begin{array}{l}64 \\
62\end{array}$ & $\begin{array}{l}4.1 \\
4.4\end{array}$ & $\begin{array}{l}1.7 \\
2.1\end{array}$ & $\begin{array}{l}1.5 \\
1.9\end{array}$ & $\begin{array}{l}0.94 \\
0.93\end{array}$ & $\begin{array}{l}>20 \\
>20\end{array}$ \\
\hline 63 & $\begin{array}{l}\text { Control } \\
\text { Aldo. }\end{array}$ & 410 & $\begin{array}{l}147 \\
160\end{array}$ & $\begin{array}{l}4.0 \\
4.0\end{array}$ & $\begin{array}{l}39 \\
36\end{array}$ & $\begin{array}{l}5.6 \\
4.8\end{array}$ & $\begin{array}{l}3.6 \\
5.8\end{array}$ & $\begin{array}{l}4.0 \\
5.0\end{array}$ & $\begin{array}{l}0.96 \\
0.96\end{array}$ & $\begin{array}{l}>20 \\
>20\end{array}$ \\
\hline 65 & $\begin{array}{l}\text { Control } \\
\text { Aldo. }\end{array}$ & 425 & $\begin{array}{l}144 \\
151\end{array}$ & $\begin{array}{l}4.5 \\
3.7\end{array}$ & $\begin{array}{l}43 \\
51\end{array}$ & $\begin{array}{l}3.2 \\
3.0\end{array}$ & $\begin{array}{l}3.8 \\
3.3\end{array}$ & $\begin{array}{l}3.3 \\
2.8\end{array}$ & $\begin{array}{l}0.95 \\
0.90\end{array}$ & $\begin{array}{l}>20 \\
>20\end{array}$ \\
\hline 66 & $\begin{array}{l}\text { Control } \\
\text { Aldo. }\end{array}$ & 380 & $\begin{array}{l}142 \\
148\end{array}$ & $\begin{array}{l}4.5 \\
4.1\end{array}$ & $\begin{array}{l}73 \\
72\end{array}$ & $\begin{array}{l}4.0 \\
4.0\end{array}$ & $\begin{array}{l}3.8 \\
2.0\end{array}$ & $\begin{array}{l}3.7 \\
2.0\end{array}$ & $\begin{array}{l}0.99 \\
0.90\end{array}$ & $\begin{array}{l}>20 \\
>20\end{array}$ \\
\hline 67 & $\begin{array}{l}\text { Control } \\
\text { Aldo. }\end{array}$ & 500 & $\begin{array}{l}143 \\
146\end{array}$ & $\begin{array}{l}4.5 \\
4.4\end{array}$ & $\begin{array}{l}45 \\
40\end{array}$ & $\begin{array}{l}4.5 \\
5.4\end{array}$ & $\begin{array}{l}4.3 \\
5.4\end{array}$ & $\begin{array}{l}4.1 \\
4.9\end{array}$ & $\begin{array}{l}0.95 \\
0.99\end{array}$ & $\begin{array}{l}>20 \\
>20\end{array}$ \\
\hline Co & $\begin{array}{l}\text { trol } \\
\text { Iean } \\
\text { D }\end{array}$ & & $\begin{array}{l}145 \\
\pm 2\end{array}$ & $\begin{array}{r}3.9 \\
\pm 0.5\end{array}$ & $\begin{array}{c}54 \\
\pm 13\end{array}$ & $\begin{array}{r}4.3 \\
\pm 0.7\end{array}$ & $\begin{array}{r}3.4 \\
\pm 0.2\end{array}$ & $\begin{array}{r}3.1 \\
\pm 0.8\end{array}$ & $\begin{array}{r}0.98 \\
\pm 0.04\end{array}$ & $>20$ \\
\hline Ald & $\begin{array}{l}\text { osterone } \\
\text { lean } \\
\text { D }\end{array}$ & & $\begin{array}{l}149 \\
\pm 7\end{array}$ & $\begin{array}{r}4.2 \\
\pm 0.9\end{array}$ & $\begin{array}{c}53 \\
\pm 14\end{array}$ & $\begin{array}{r}4.4 \\
\pm 1.0\end{array}$ & $\begin{array}{r}3.8 \\
\pm 1.9\end{array}$ & $\begin{array}{r}3.2 \\
\pm 1.8\end{array}$ & $\begin{array}{r}0.95 \\
\pm 0.7\end{array}$ & $>20$ \\
\hline $\begin{array}{r}\text { Dif } \\
1 \\
F\end{array}$ & $\begin{array}{l}\text { erence } \\
\text { lean }\end{array}$ & & +4 & $\begin{array}{l}+0.2 \\
>0.20\end{array}$ & $\begin{array}{l}-0.8 \\
>0.50\end{array}$ & $\begin{array}{l}+0.1 \\
>0.50\end{array}$ & $\begin{array}{l}+0.3 \\
>0.50\end{array}$ & $\begin{array}{l}+1.0 \\
+0.1 \\
>0.50\end{array}$ & $\begin{array}{l}-0.3 \\
>0.20\end{array}$ & \\
\hline
\end{tabular}


TABLE III

The effects of blocking endogenous aldosterone activity with spirolactones on $\mathrm{T}^{\mathrm{c}} \mathrm{H}_{2} \mathrm{O}$ formation during mannitol diuresis in sodium-depleted dogs

\begin{tabular}{|c|c|c|c|c|c|c|c|c|}
\hline Dog & Preparation, no. of days & $\underset{\mathrm{Na}}{\text { Serum }}$ & $\underset{\mathbf{K}}{\text { Serum }}$ & GFR & 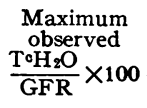 & $\begin{array}{c}\text { Derived } \\
\frac{T^{\circ} \mathrm{H}_{2} \mathrm{O}}{\mathrm{GFR}} \times 100\end{array}$ & Slope & $\begin{array}{c}\text { Calculated } \\
\text { point of } \\
\text { crossover }\end{array}$ \\
\hline & & $m E q / L$ & $m E q / L$ & $\mathrm{ml} / \mathrm{min}$ & $\begin{array}{l}m l / 100 \\
m l G F R\end{array}$ & 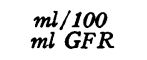 & & $\mathrm{ml} / \mathrm{min}$ \\
\hline \multirow[t]{2}{*}{53} & \multirow{2}{*}{$\begin{array}{l}\text { Na-free, } 8 \\
\text { Na-free, } 15 ;+ \\
\quad \text { oral SC-9420, } 7\end{array}$} & 140 & 3.6 & 59 & 1.7 & 1.2 & 0.86 & 11.4 \\
\hline & & 133 & 3.8 & 45 & 3.5 & 2.5 & 0.88 & 15.8 \\
\hline \multirow[t]{2}{*}{62} & \multirow{2}{*}{$\begin{array}{l}\text { Na-fed control } \\
\text { Na-free, } 7 \\
\text { Na-free, } 14 ;+ \\
\quad \text { SC-11927, } 10 \mathrm{mg}, \text { i.m., } \\
\quad\end{array}$} & $\begin{array}{l}149 \\
143\end{array}$ & $\begin{array}{l}3.8 \\
4.7\end{array}$ & $\begin{array}{l}64 \\
60\end{array}$ & $\begin{array}{l}1.7 \\
0.5\end{array}$ & $\begin{array}{l}1.5 \\
0.6\end{array}$ & $\begin{array}{l}0.94 \\
0.97\end{array}$ & $\begin{array}{l}>20 \\
16.7\end{array}$ \\
\hline & & 138 & 4.7 & 59 & 2.0 & 0.3 & 0.64 & 7.8 \\
\hline \multirow[t]{2}{*}{66} & \multirow{2}{*}{$\begin{array}{l}\text { Na-fed control } \\
\text { Na-free, } 7 \\
\text { Na-free, } 14 ;+ \\
\quad \text { SC-11927, } 10 \mathrm{mg}, \text { i.m., }\end{array}$} & 142 & 4.5 & $\cdot 73$ & 3.8 & 3.7 & 0.99 & $>20$ \\
\hline & & 143 & 4.9 & 52 & 1.5 & -0.4 & 0.70 & 8.0 \\
\hline \multirow[t]{2}{*}{69} & \multirow{2}{*}{$\begin{array}{l}\text { Na-fed control } \\
\text { Na-free, } 10 \\
\text { Na-free, } 17 ;+ \\
\quad \text { SC-11927, } 20 \mathrm{mg} \text {, i.m., }\end{array}$} & $\begin{array}{l}145 \\
146\end{array}$ & $\begin{array}{l}4.1 \\
4.0\end{array}$ & $\begin{array}{l}47 \\
42\end{array}$ & $\begin{array}{l}4.6 \\
3.3\end{array}$ & $\begin{array}{l}3.7 \\
2.0\end{array}$ & $\begin{array}{l}0.99 \\
0.74\end{array}$ & $\begin{array}{c}>20 \\
11.2\end{array}$ \\
\hline & & 149 & 4.4 & 48 & 4.8 & 2.0 & 0.84 & 14.2 \\
\hline \multirow[t]{2}{*}{39} & \multirow{2}{*}{$\begin{array}{l}\text { Na-fed control } \\
\text { Na-free, } 10 \\
\text { Na-free, } 16 ;+ \\
\quad \text { SC-11927, } 20 \mathrm{mg}, \text { i.m., } \\
\quad 6\end{array}$} & $\begin{array}{l}147 \\
143\end{array}$ & $\begin{array}{l}3.8 \\
4.4\end{array}$ & $\begin{array}{l}72 \\
54\end{array}$ & $\begin{array}{l}5.8 \\
4.5\end{array}$ & $\begin{array}{l}5.0 \\
0.3\end{array}$ & $\begin{array}{l}1.12 \\
0.81\end{array}$ & $\begin{array}{l}>20 \\
12.3\end{array}$ \\
\hline & & 140 & 4.7 & 44 & 2.6 & 0.8 & 0.87 & 13.9 \\
\hline \multirow[t]{3}{*}{71} & \multirow{3}{*}{$\begin{array}{l}\text { Na-fed control } \\
\text { Na-free, } 8 \\
\text { Na-free, } 14 ;+ \\
\quad \text { SC-11927, } 100 \text { mg, i.m., } \\
\quad 6\end{array}$} & 146 & 3.7 & 64 & 5.6 & 4.8 & 1.07 & $>20$ \\
\hline & & 145 & 4.5 & 44 & 2.0 & 0.1 & 0.76 & 6.9 \\
\hline & & 136 & 5.0 & 45 & & 0.8 & 0.89 & 7.3 \\
\hline
\end{tabular}

C. Aldosterone and aldosterone antagonists. Sodium restriction is known to cause a rise in endogenous aldosterone secretion. If aldosterone caused a significant degree of sodium reabsorption in the proximal tubule, it might, by making less sodium available for reabsorption in the ascending limb of the loop of Henle, be responsible for the impairment in urinary concentration observed in sodium deprivation.

To examine this hypothesis, ten dogs were maintained on the sodium-containing diet. Studies were performed after 3 to 5 days and were then repeated 4 to 7 days later. Two hours before the repeat study, 30 to $500 \mu \mathrm{g}$ of DL-aldosterone per $\mathrm{kg}$ body weight was given i.v. The results are summarized in Table II. No significant changes in GFR, slope, $\mathrm{T}_{\mathrm{M}} \mathrm{H}_{2} \mathrm{O}$, maximum osmotic U/P ratio, serum sodium and serum potassium were observed. One typical study is shown in Figure
3. The activity of the aldosterone preparation was attested to by a decreased sodium excretion rate and an increased potassium excretion rate at any given urine flow when compared with the control study in the same dog. It appeared then that exogenous aldosterone alone was incapable of diminishing $\mathrm{T}^{\mathrm{c}} \mathrm{H}_{2} \mathrm{O}$ formation.

To explore the possibility that excessive endogenous aldosterone, when coupled with diminished filtered sodium, might still be an essential component in depressing $\mathrm{T}^{\mathrm{c}} \mathrm{H}_{2} \mathrm{O}$, further studies were done. Five sodium-restricted dogs displaying typical defective urinary concentrating ability were continued on sodium deprivation and given an aldosterone antagonist for 7 days. The results (Table IIII) show that by blocking the action of aldosterone, neither $\mathrm{T}^{\mathrm{c}} \mathrm{H}_{2} \mathrm{O}$ formation, maximum urinary concentrating ability, nor GFR was restored to normal (Figure 4). 


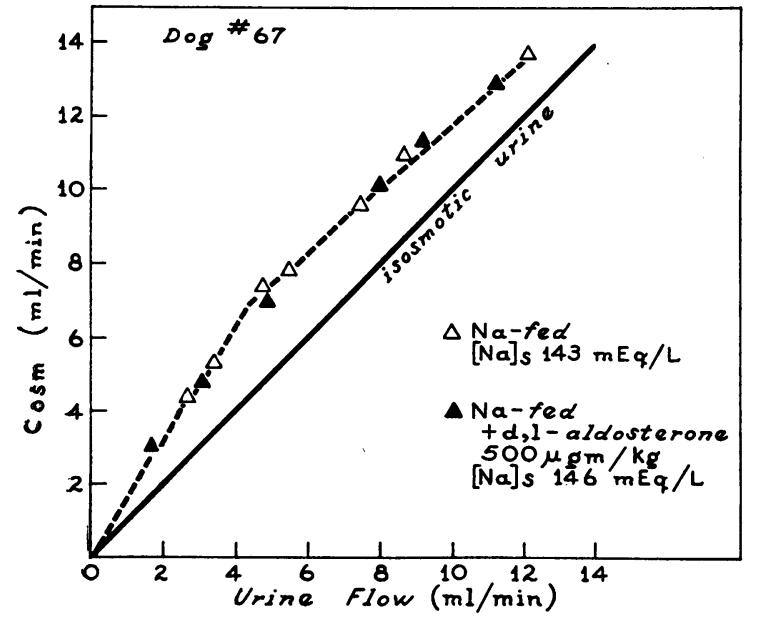

Fig. 3. ThE EFFECT OF EXOGENOUS ALDOSTERONE ON $\mathrm{T}^{\mathrm{c}} \mathrm{H}_{2} \mathrm{O}$ FORMATION DURING MANNITOL DIURESIS IN A SODIUM-FED DOG.

It seems that the defect in renal water conservation produced by sodium restriction is not due to increased endogenous aldosterone secretion.

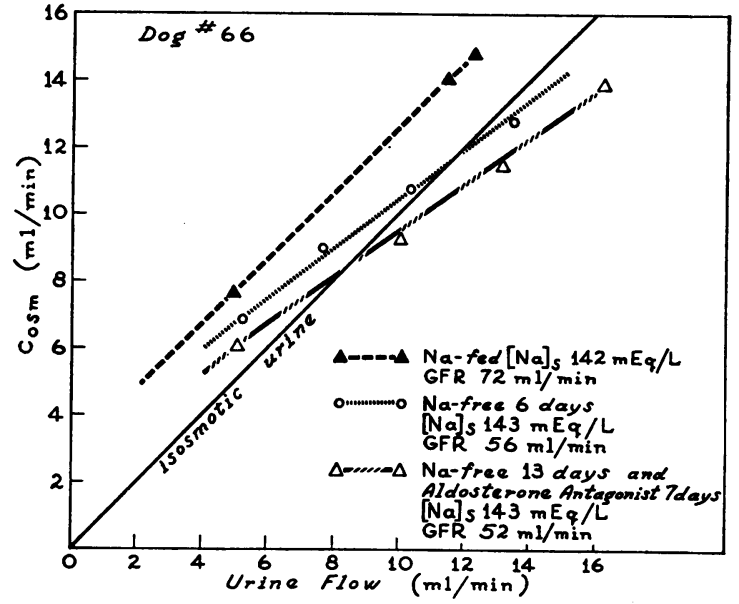

Fig. 4. THE EFFECT OF BLOCKING ENDOGENOUS ALDOSTERONE ACTIVITY WITH SC-11927 ON $\mathrm{T}^{\mathrm{c}} \mathrm{H}_{2} \mathrm{O}$ FORMATION DURING MANNITOL DIURESIS IN A SODIUM-DEPLETED DOG.

D. Raising GFR during sodium restriction. Inasmuch as the defect in urinary concentration was displayed only when GFR had fallen below control levels, the effect of increasing GFR in the

TABLE IV

The effects of increasing GFR with methylprednisolone on $T^{\circ} \mathrm{H}_{2} \mathrm{O}$ formation during mannitol diuresis in sodium-depleted dogs

\begin{tabular}{|c|c|c|c|c|c|c|c|c|}
\hline Dog & Preparation, no. of days & $\underset{\mathrm{Na}}{\text { Serum }}$ & $\underset{\mathbf{K}}{\text { Serum }}$ & GFR & $\begin{array}{c}\underset{\text { Maximum }}{\text { observed }} \\
\frac{\mathrm{T}_{2} \mathrm{O}}{\mathrm{GFR}} \times 100\end{array}$ & 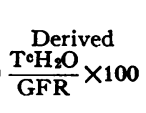 & Slope & $\begin{array}{l}\text { Calculated } \\
\text { point of } \\
\text { crossover }\end{array}$ \\
\hline & & $m E q / L$ & $m E q / L$ & $m l / m i n$ & $\begin{array}{l}m l / 100 \\
m l G F R\end{array}$ & $\begin{array}{l}m l / 100 \\
m l G F R\end{array}$ & & $\operatorname{ml} / \min$ \\
\hline \multirow[t]{2}{*}{43} & \multirow{2}{*}{$\begin{array}{l}\text { Na-fed control } \\
\text { Na-free, } 3 \\
\text { Na-free, } 7 ;+ \text { acute } \\
\quad \text { methylprednisolone }\end{array}$} & $\begin{array}{l}149 \\
144\end{array}$ & $\begin{array}{l}4.3 \\
4.6\end{array}$ & $\begin{array}{l}56 \\
37\end{array}$ & $\begin{array}{l}5.3 \\
1.3\end{array}$ & $\begin{array}{r}4.4 \\
-0.2\end{array}$ & $\begin{array}{l}0.95 \\
0.74\end{array}$ & $>20$ \\
\hline & & 145 & 3.9 & 54 & 3.3 & 1.9 & 0.84 & 12.5 \\
\hline \multirow[t]{2}{*}{47} & \multirow{2}{*}{$\begin{array}{l}\text { Na-fed control } \\
\text { Na-free, } 7 \\
\text { Na-free, } 7 ;+ \\
\text { methylprednisolone, } \\
\quad 6\end{array}$} & $\begin{array}{l}149 \\
152\end{array}$ & $\begin{array}{l}4.5 \\
5.3\end{array}$ & $\begin{array}{l}81 \\
66\end{array}$ & $\begin{array}{l}1.6 \\
0.8\end{array}$ & $\begin{array}{r}1.4 \\
-0.8\end{array}$ & $\begin{array}{l}1.02 \\
0.96\end{array}$ & $\begin{array}{l}>20 \\
<1.0\end{array}$ \\
\hline & & 145 & 3.5 & 105 & 2.5 & 1.7 & 0.84 & $>20$ \\
\hline \multirow[t]{2}{*}{48} & \multirow{2}{*}{$\begin{array}{l}\text { Na-fed control } \\
\text { Na-free, } 9 \\
\text { Na-free, } 7 ;+ \\
\quad \text { methylprednisolone, } \\
6\end{array}$} & $\begin{array}{l}148 \\
144\end{array}$ & $\begin{array}{l}4.5 \\
4.8\end{array}$ & $\begin{array}{l}50 \\
44\end{array}$ & $\begin{array}{l}6.5 \\
2.3\end{array}$ & $\begin{array}{l}4.8 \\
1.2\end{array}$ & $\begin{array}{l}1.06 \\
0.99\end{array}$ & $\begin{array}{l}>20 \\
12.2\end{array}$ \\
\hline & & 140 & 4.1 & 59 & 5.6 & 4.7 & 1.10 & $>20$ \\
\hline \multirow[t]{3}{*}{67} & \multirow{3}{*}{$\begin{array}{l}\text { Na-fed control } \\
\text { Na-free, } 6 \\
\text { Na-free, } 9 ;+ \\
\quad \text { methylprednisolone, } \\
\quad 4\end{array}$} & 143 & 4.5 & 45 & 4.3 & 4.1 & 0.95 & $>20$ \\
\hline & & 141 & 4.0 & 41 & 1.6 & 1.3 & 0.89 & 9.1 \\
\hline & & 135 & 4.1 & 50 & 4.0 & 4.0 & 1.03 & $>20$ \\
\hline \multirow[t]{2}{*}{62} & \multirow{2}{*}{$\begin{array}{l}\text { Na-fed control } \\
\text { Na-free, } 14 \\
\text { Na-free, } 16 ;+ \\
\quad \text { methylprednisolone, } \\
\quad 4\end{array}$} & $\begin{array}{l}149 \\
138\end{array}$ & $\begin{array}{l}3.8 \\
4.7\end{array}$ & $\begin{array}{l}64 \\
59\end{array}$ & $\begin{array}{l}1.7 \\
2.0\end{array}$ & $\begin{array}{l}1.5 \\
0.3\end{array}$ & $\begin{array}{l}0.94 \\
0.64\end{array}$ & $\begin{array}{r}>20 \\
7.8\end{array}$ \\
\hline & & 150 & 4.3 & 64 & 6.2 & 5.5 & 1.04 & $>20$ \\
\hline
\end{tabular}


face of sodium restriction was examined. Five dogs, displaying the usual defect on sodium restriction, were continued on the sodium-free diet and given methylprednisolone. In all five there was an elevation of GFR to or above control levels (Table IV). In four experiments $\mathrm{T}_{\mathbf{M}} \mathrm{H}_{2} \mathrm{O}$ reached values at or close to those of their controls, but in one the restitution was only to about half normal. In this animal (Dog 43), the methylprednisolone was given i.v. only 20 minutes prior to study, whereas in those with complete correction it had been given for several days. One study is shown in Figure 5.

Methylprednisolone was chosen as the agent to increase filtration rate because it has negligible mineralocorticoid activity. Although it has been postulated that glucocorticoid inhibits back diffusion of water in the renal tubule (18), this effect would serve only to enhance the defect rather than correct it. Attempts to raise GFR in the saltdeprived dogs with dextran, aminophylline, and glycine infusions were unsuccessful.

E. Hyponatremia. The defect in urinary con-

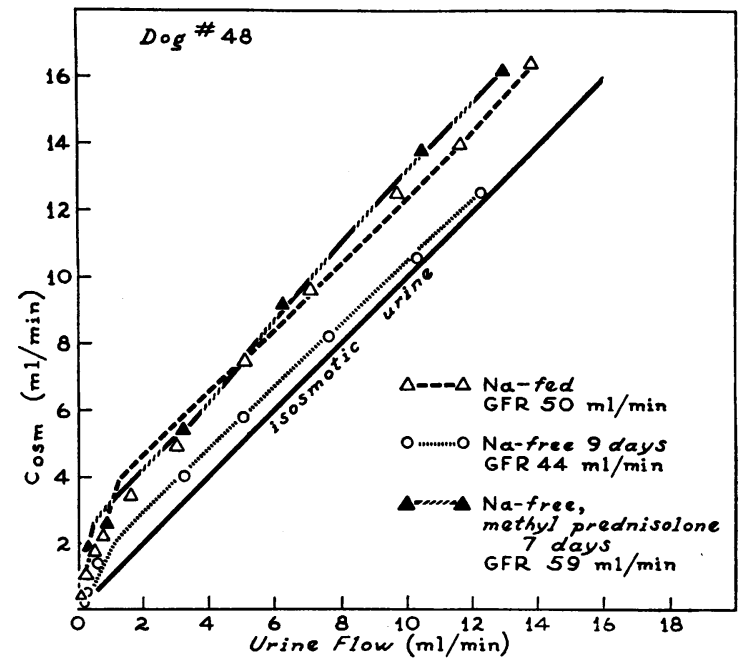

Fig. 5. The effect of RaIsing GFR with MethylPREDNISOLONE ON $\mathrm{T}^{\mathrm{c}} \mathrm{H}_{2} \mathrm{O}$ FORMATION DURING MANNITOL DIURESIS IN A SODIUM-DEPLETED DOG.

centration during sodium deprivation appears to be intimately related to a reduction in GFR. Whether or not this is simply the consequence of a diminished filtered load of sodium was explored

TABLE $\mathrm{V}$

The effects of hyponatremia on $\mathrm{T}^{ } \mathrm{H}_{2} \mathrm{O}$ formation during mannitol diuresis in sodium-fed and sodium-depleted dogs

\begin{tabular}{|c|c|c|c|c|c|c|c|c|c|}
\hline Dog & Preparation, no. of days & $\underset{\mathrm{Na}}{\text { Serum }}$ & $\underset{\mathbf{K}}{\text { Serum }}$ & GFR & $\begin{array}{l}\text { Maximum } \\
\text { osmotic } T \\
\text { U/P ratio }\end{array}$ & 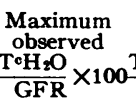 & 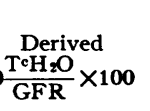 & Slope & $\begin{array}{c}\text { Calculated } \\
\text { point of } \\
\text { crossover }\end{array}$ \\
\hline \multirow{4}{*}{12} & \multirow{4}{*}{$\begin{array}{l}\text { Na-free, } 8 \\
\text { Na-free, } 11 ;+ \\
\quad \text { vasopressin } \\
\text { Na-free, } 14 ;+ \\
\quad \text { vasopressin }+\mathrm{H}_{2} \mathrm{O}\end{array}$} & $m E q / L$ & $m E q / L$ & $m l / m i n$ & & $\begin{array}{l}m l / 100 \\
m l / G F R\end{array}$ & $\begin{array}{l}m l / 100 \\
m l / G F R\end{array}$ & & $m l / m i n$ \\
\hline & & 152 & 4.4 & 38 & 3.0 & 1.9 & -1.5 & 0.81 & 4.2 \\
\hline & & 149 & 4.3 & 46 & 3.3 & 2.7 & -0.3 & 0.74 & 6.5 \\
\hline & & 120 & 4.8 & 42 & 1.7 & 2.0 & -1.1 & 0.80 & 5.0 \\
\hline \multirow[t]{3}{*}{23} & \multirow{3}{*}{$\begin{array}{l}\text { Na-fed control } \\
\text { Na-fed + vasopressin } \\
\text { Na-fed + vasopressin } \\
\quad+\mathrm{H}_{2} \mathrm{O}\end{array}$} & 149 & 3.1 & 40 & 2.7 & 5.2 & 3.2 & 0.94 & $>20$ \\
\hline & & 145 & 4.0 & 42 & 3.4 & 8.4 & 6.2 & 1.10 & $>20$ \\
\hline & & 126 & 4.1 & 41 & 2.3 & 5.2 & 4.5 & 1.02 & $>20$ \\
\hline \multirow[t]{2}{*}{58} & \multirow{2}{*}{$\begin{array}{l}\mathrm{Na} \text {-fed control } \\
\mathrm{Na} \text {-fed }+ \text { vasopressin } \\
\quad+\mathrm{H}_{2} \mathrm{O}\end{array}$} & 159 & 3.9 & 53 & 5.5 & 4.8 & 3.8 & 1.07 & $>20$ \\
\hline & & 126 & 4.2 & 48 & 4.6 & 3.6 & 2.9 & 0.95 & $>20$ \\
\hline \multirow[t]{3}{*}{68} & \multirow{3}{*}{$\begin{array}{l}\mathrm{Na}-\mathrm{fed} \text { control } \\
\mathrm{Na}-\mathrm{fed}+\text { vasopressin } \\
+\mathrm{H}_{2} \mathrm{O} \\
\mathrm{Na-free}, 4 ;+ \\
\quad \text { vasopressin }\end{array}$} & 149 & 3.9 & 59 & 3.8 & 6.8 & 5.9 & 1.05 & $>20$ \\
\hline & & 128 & 3.4 & 63 & 5.6 & 6.3 & 5.3 & 1.06 & $>20$ \\
\hline & & 117 & 3.6 & 56 & 4.8 & 5.1 & 4.9 & 0.73 & 16.4 \\
\hline \multirow[t]{4}{*}{69} & \multirow{4}{*}{$\begin{array}{l}\mathrm{Na} \text {-fed control } \\
\mathrm{Na}-\text { fed }+ \text { vasopressin } \\
\quad+\mathrm{H}_{2} \mathrm{O} \\
\mathrm{Na}-\mathrm{free}, 10 \\
\mathrm{Na} \text {-free, } 4 ;+ \\
\quad \text { vasopressin }\end{array}$} & 145 & 4.1 & 47 & 2.5 & 4.6 & 3.7 & 0.99 & $>20$ \\
\hline & & 121 & 3.5 & 47 & 3.4 & 4.9 & 3.8 . & 0.98 & $>20$ \\
\hline & & 146 & 4.0 & 42 & 3.0 & 3.3 & 2.0 & 0.74 & ' 11.2 \\
\hline & & 120 & 3.8 & 41 & 2.1 & 2.8 & -1.4 & 0.59 & 6.6 \\
\hline
\end{tabular}



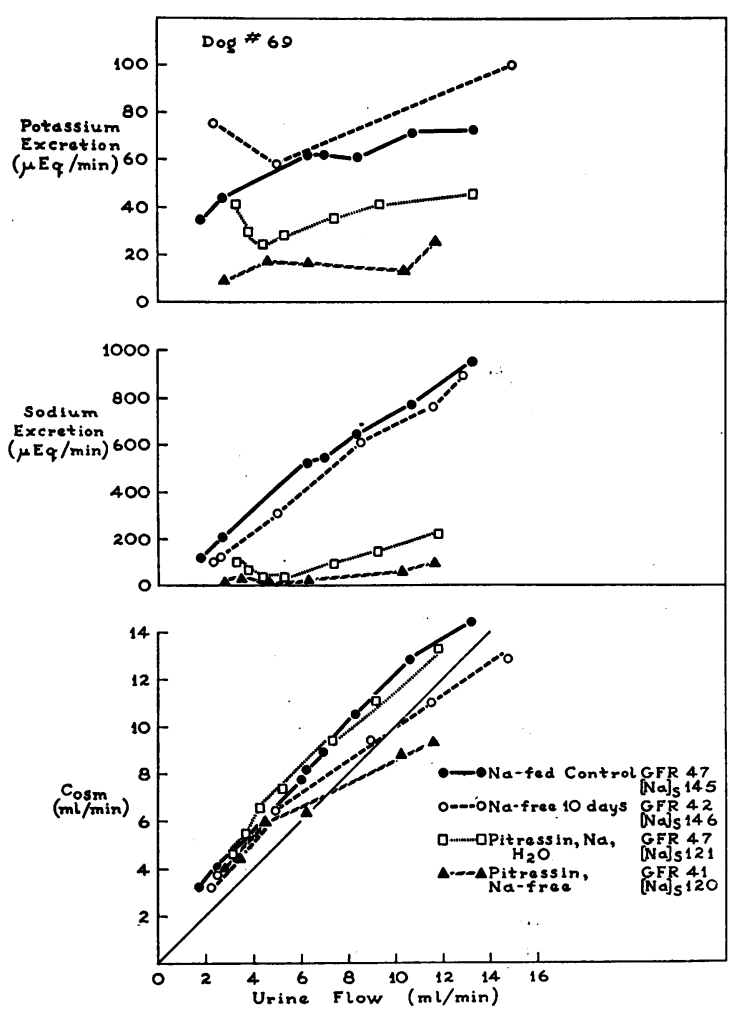

Fig. 6. Variations in $\mathrm{T}^{\mathrm{c}} \mathrm{H}_{2} \mathrm{O}$ formation, sodium EXCRETION, AND POTASSIUM EXCRETION DURING MANNITOL DIURESIS IN A DOG SODIUM-FED AND THEN SODIUM-DEPRIVED WITH NORMAL AND LOW LEVELS OF SERUM SODIUM.

in another group of studies. In these studies filtered sodium was diminished by inducing hyponatremia with chronic vasopressin administration and excess water. Filtered sodium was reduced $13,28,8$, and 17 per cent (average, 17 per cent) in these four hyponatremic dogs (Table V) compared with an average 23 per cent reduction in filtered sodium in the salt-restricted dogs. Despite comparable reduction of filtered sodium during hyponatremia there was no change in $\mathrm{T}^{\mathrm{c}} \mathrm{H}_{2} \mathrm{O}$ formation. In Dog 69 (Figure 6) a 17 per cent reduction in filtered sodium during hyponatremia had no effect on $\mathrm{T}^{\mathrm{c}} \mathrm{H}_{2} \mathrm{O}$ formation, whereas a 10 per cent reduction in filtered sodium during salt deprivation drastically reduced $\mathrm{T}^{\mathrm{c}} \mathrm{H}_{2} \mathrm{O}$ formation. Hence it is concluded that diminished filtered sodium per se does not reproduce the defect in $\mathrm{T}^{\mathrm{c}} \mathrm{H}_{2} \mathrm{O}$ formation observed in salt-restricted dogs.

\section{DISCUSSION}

Sodium restriction in dogs impairs urinary concentration in an unusual fashion: the ability to produce maximally concentrated urine is only slightly impaired, whereas the formation of $\mathrm{T}^{\mathrm{c}} \mathrm{H}_{2} \mathrm{O}$ is curtailed to the extent that hypotonic urine is excreted at moderate urine flows. Thus salt deprivation profoundly alters the normal function of the renal concentrating mechanism.

According to current concepts of urine concentration, distal tubular fluid equilibrates to isotonicity under the influence of antidiuretic hormone before entering the collecting tubule, even during severe mannitol diuresis (3). In the collecting tubule water is abstracted along osmotic gradients into the hypertonic medullary interstitium, resulting in $\mathrm{T}^{\mathrm{c}} \mathrm{H}_{2} \mathrm{O}$ formation and the elaboration of a hypertonic urine. Any factor, therefore, that interferes with medullary hypertonicity will alter both the ability to produce maximally concentrated urine and $\mathrm{T}^{\mathrm{c}} \mathrm{H}_{2} \mathrm{O}$ formation. However, a reduction in medullary hypertonicity alone will not result in the formation of hypotonic urine. Since there is no convincing evidence that the collecting tubule is capable of reabsorbing solute in excess of water during either diuresis or antidiuresis, the excretion of a hypotonic urine is prima facie evidence that the fluid entering the collecting tubule is hypotonic. ${ }^{4}$

A fall in medullary hypertonicity, however, could result in excretion of hypotonic urine if, in the dog (in contrast to the rat), fluid issuing from the distal tubule were always hypotonic. In the presence of $\mathrm{ADH}$ activity, the delivery of hypotonic urine to the collecting tubule would normally be obscured by extraction of large amounts of $\mathrm{T}^{\mathrm{c}} \mathrm{H}_{2} \mathrm{O}$. If this were the case, impairment of medullary hypertonicity during salt deprivation could in fact result in hypotonic urine in mannitol diuresis. Although most investigators have assumed that in the presence of ADH the fluid leaving the distal tubule is always isotonic in man, dog and rat, only in the rat is direct micropuncture evidence available to establish this point (3). The fact that man and dog excrete hypotonic urine in the presence of maximum antidiuretic hormone in certain experimental and pathological condi-

${ }^{4}$ Wirz (19) has published observations on six water diureses: the osmotic pressure of ureteral urine was higher than that of the distal tubule in one instance, the same in two instances, and slightly lower in three. Indeed, Gottschalk and Mylle (3) have shown that the osmotic pressure of ureteral urine is higher than that of the distal tubule in diabetes insipidus. 
tions $(4,6,9,13,16,20-22)$ while the rat, in these same conditions, shows only decreased urinary concentration but never hypotonic urine, suggests that the equilibration of fluid in the distal tubule may vary between these species.

If, then, in the dog hypotonic fluid were always entering the collecting tubule the concentrating defect of sodium restriction could be entirely explained by a decrease in medullary hypertonicity. A possible mechanism by which sodium deprivation might reduce medullary hypertonicity is a reduced delivery of sodium to the loop of Henle, as a result of either increased proximal tubular reabsorption due to enhanced aldosterone secretion or diminished sodium loads as a result of a fall in GFR. The failure of aldosterone in normal dogs to produce, and spirolactone in sodium-restricted dogs to correct the defect is strong evidence that augmented aldosterone secretion is not responsible for the impaired urinary concentrating ability. The only factor in these studies that was consistently related to the defect was a chronic reduction in GFR. The fact that administration of methylprednisolone to salt-restricted dogs both raised GFR and restored the capacity to form $\mathrm{T}^{\mathrm{c}} \mathrm{H}_{2} \mathrm{O}$ suggests that a reduction in GFR is in some manner implicated in the observed defect in urinary concentration.

A reduction in GFR, by diminishing the amount of filtered sodium, might result in impaired urinary concentration by reducing the delivery of sodium to the loop of Henle. In the hyponatremic dogs, however, the reduction in filtered sodium, comparable in degree with that produced by sodium restriction, failed to impair concentrating ability. It therefore seems unlikely that the amount of filtered sodium is the determining factor.

It is conceivable, however, that when filtered sodium is reduced via a lowered GFR, less sodium reaches the loop of Henle than when a similar reduction in filtered sodium is induced by hyponatremia. This possibility was examined by comparing the excretion of sodium and potassium during mannitol diuresis in hyponatremic and salt-restricted dogs (Figure 6). In salt-deprived dogs with normal serum sodium the same amount of sodium was excreted at any given urine flow as in sodium-fed dogs, suggesting that during salt deprivation normal amounts of sodium reach the loop of Henle. In contrast, sodium and potas- sium excretion are much less at comparable urine flows in hyponatremic dogs. The reduction in potassium excretion in these dog suggests that less sodium traverses the loop of Henle to reach the distal tubule. Therefore, despite the fact that normal amounts of sodium pass through the loop in the sodium-deprived dogs, $\mathrm{T}^{\mathrm{c}} \mathrm{H}_{2} \mathrm{O}$ formation is defective. On the other hand, reduced amounts of sodium leave the loop of Henle in both the sodium-fed and sodium-depleted hyponatremic dog; however, $\mathrm{T}^{\mathrm{c}} \mathrm{H}_{2} \mathrm{O}$ is markedly impaired in hyponatremic dogs deprived of sodium but normal in those that are sodium-fed. Therefore the reduction in GFR, that appears to be implicated in defective $\mathrm{T}^{\mathrm{c}} \mathrm{H}_{2} \mathrm{O}$ formation in sodium deprivation, does not appear to mediate this defect by reducing the delivery of sodium to the loop of Henle. It is not clear, therefore, how a reduced GFR could result in a decrease in medullary hypertonicity. Moreover, the observation that the maximum osmotic $\mathrm{U} / \mathrm{P}$ ratio is only slightly altered by sodium restriction suggests that reduction in medullary hypertonicity is not the mechanism involved.

If, on the other hand, the dog is like the rat in that distal tubular fluid normally equilibrates to isotonicity before entering the collecting tubule, no degree of impairment of medullary hypertonicity could explain the excretion of hypotonic urine, and another mechanism must be involved. An alternative explanation is that sodium deprivation in some ways impairs the permeability of the distal tubule to water and thus prevents equilibration of distal tubular fluid to isotonicity. Under these circumstances mannitol diuresis would sweep increasing quantities of hypotonic fluid into the collecting tubule, thus obscuring $\mathrm{T}^{\mathrm{c}} \mathrm{H}_{2} \mathrm{O}$ formation and resulting in excretion of hypotonic urine unrelated to either sodium transport by the loop of Henle or medullary tonicity. The manner in which sodium depletion could alter the permeability of the distal tubule in this fashion is not clear.

SUM MARY

Urinary concentrating ability was studied in dogs before and after sodium restriction by measuring maximum urinary concentration and $\mathrm{T}^{\mathrm{c}} \mathrm{H}_{2} \mathrm{O}$ formation during. mannitol diuresis. Sodium deprivation resulted in a slight fall in maximum osmotic $\mathrm{U} / \mathrm{P}$ ratios and a marked fall in $\mathrm{T}^{\mathrm{c}} \mathrm{H}_{2} \mathrm{O}$ 
formation so that hypotonic urine was excreted at moderate urine flows. This alteration in urinary concentrating ability was not due to an increased aldosterone secretion, since exogenous aldosterone failed to produce it in sodium-fed dogs and aldosterone antagonists failed to correct it in sodium-deprived dogs. The defect, however, was always associated with a fall in glomerular filtration rate (GFR) and was corrected by raising GFR in salt-restricted dogs with methylprednisolone. That diminished GFR did not produce the defect by decreasing the amount of sodium reaching the loop of Henle was shown by the ability of sodium-fed hyponatremic dogs to form normal $\mathrm{T}^{\mathrm{c}} \mathrm{H}_{2} \mathrm{O}$ despite a reduction in sodium delivery to the loop of Henle to levels far below those of normonatremic sodium-deprived dogs. It is concluded that the defect in urinary concentration seen in sodium deprivation is due to the failure of distal tubular fluid to equilibrate to isotonicity before reaching the collecting tubule.

\section{REFERENCES}

1. Wirz, $\mathrm{H}$. The production of hypertonic urine by the mammalian kidney in Ciba Foundation Symposium on The Kidney, A. A. G. Lewis and G. E. W. Wolstenholme, Ed. Boston, Little, Brown, 1954, p. 38.

2. Berliner, R. W., Levinsky, N. G., Davidson, D. G., and Eden, $M$. Dilution and concentration of the urine and the action of antidiuretic hormone. Amer. J. Med. 1958, 24, 730.

3. Gottschalk, C. W., and Mylle, M. Micropuncture study of the mammalian urinary concentrating mechanism: Evidence for the countercurrent hypothesis. Amer. J. Physiol. 1959, 196, 927.

4. Levinsky, N. G., Davidson, D. G., and Berliner, R. W. Changes in urine concentration during prolonged administration of vasopressin and water. Amer. J. Physiol. 1959, 196, 451.

5: Levitt; M. F., Levy, M. S., and Polimeros, D. The effect of a fall in filtration rate on solute and water excretion in hydropenic man. J. clin. Invest. 1959, 38, 463.

6. Giebisch, G., and Lozanö, R. The effects of adrenal steroids and potassium depletion on the elaboration of an osmotically concentrated urine. J. clin. Invest. 1959, 38, 843.

7. Malvin; R. L., and Wilde, W. S. Washout of renal countercurrent $\mathrm{Na}$ gradient by osmotic diuresis. - Amer. J. Physiol. 1959, 197, 177.
8. Schreiner, G. E. Determination of inulin by means of resorcinol. Proc. Soc. exp. Biol. (N. Y.) 1950, 74, 117.

9. Zak, G. A., Brun, C., and Smith, H. W. The mechanism of formation of osmotically concentrated urine during the antidiuretic state. J. clin. Invest. 1954, 33, 1064.

10. Snedecor, G. W. Statistical Methods Applied to Experiments in Agriculture and Biology, 5th ed. Ames, Iowa State College Press, 1956.

11. Boyarsky, S., and Smith, H. W. Renal concentrating operation at low urine flows. J. Urol. (Baltimore) 1957, 78, 511.

12. Page, L. B., and Reem, G. H. Urinary concentrating mechanism in the dog. Amer. J. Physiol. 1952, 171, 572.

13. Epstein, F. H., Beck, D., Carone, F. A., Levitin, H., and Manitius, $A$. Changes in renal concentrating ability produced by parathyroid extract. J. clin. Invest. 1959, 38, 1214.

14. Beck, D., Levitin, H., and Epstein, F. H. Effect of intravenous infusions of calcium on renal concentrating ability. Amer. J. Physiol. 1959, 197, 1118.

15. Epstein, F. H., Kleeman, C. R., Pursel, S., and Hendrikx, A. The effect of feeding protein and urea on the renal concentrating process. J. clin. Invest. 1957, 36, 635.

16. Levinsky, N. G., and Berliner, R. W. The role of urea in the urine concentrating mechanism. J. clin. Invest. 1959, 38, 741.

17. Raisz, L. G., Au, W. Y. W., and Scheer, R. L. Studies on the renal concentrating mechanism. IV. Osmotic diuresis. J. clin. Invest. 1959, 38, 1725.

18. Kleeman, C. R., Maxwell, M. H., and Rockney, R. E. Mechanisms of impaired water excretion in adrenal and pituitary insufficiency. I. The role of altered glomerular filtration rate and solute excretion. J. clin. Invest. 1958, 37, 1799.

19. Wirz, H. Der osmotioche Druck in den corticolen Tubuli der Rattenniere. Helv. physiol. pharmacol. Acta 1956, 14, 353.

20. Zeffren, J. L., and Heinemann, H. O. The effects of hypercalcemia on the renal concentrating mechanism in man (abstract). J. clin. Invest. 1960, 39, 1042.

21. Levinsky, N. G., Davidson, D. G., and Berliner, R. W. Effects of reduced glomerular filtration on urine concentration in the presence of antidiuretic hormone. J. clin. Invest. 1959, 38, 730.

22. Gill, J. R., Jr., and Bartter, F. C. On the impairment of renal concentrating ability in prolonged hypercalcemia and hypercalciuria in man. J. clin. Invest. $1961,40,716$. 